\title{
The Evaluation Criteria in THE RoAd TRANSPORTED WITH FUZZY LOGIC SUPPORT
}

\author{
Jan Mrazek, Jan Vavra \& Martin Hromada
}
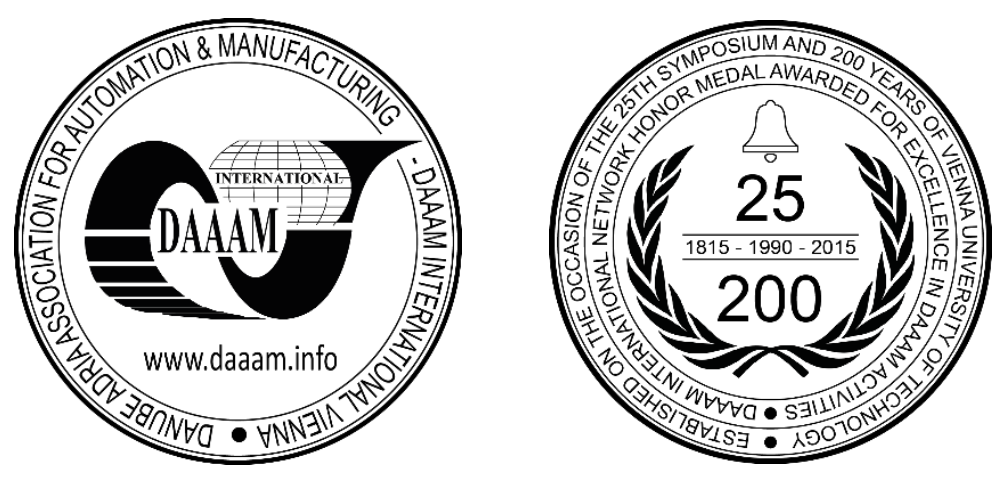

This Publication has to be referred as: Mrazek, J[an]; Vavra, J[an] \& Hromada, M[artin] (2018). The Evaluation Criteria in the Road Transported With Fuzzy Logic Support, Proceedings of the 29th DAAAM International Symposium, pp.1187-1190, B. Katalinic (Ed.), Published by DAAAM International, ISBN 978-3-902734-20-4, ISSN 1726-9679, Vienna, Austria

DOI: $10.2507 / 29$ th.daaam.proceedings. 171

\begin{abstract}
This paper addresses the issue of critical infrastructure protection regarding prevention and response when an incident of road transport. Critical infrastructure is necessary for each country. Security functionality and security of the individual components or system components. When an incident occurs always, distort proper operation. As a result of the incident sends requests to the criteria just before the onset but also in the development we must take into account. Evaluation criteria are important parts not only for prevention of occurrence of the incident as well as reaction. Implications of evaluation criteria are to minimize the risks and consequences of the incident.
\end{abstract}

Keywords: critical infrastructure; criteria of evaluation; transportation; traffic

\section{Introduction}

The critical infrastructure is important for each state or republic in the world. The critical infrastructure is defined by different sectoral criteria. These criteria are helpful for solving incidents. The preventive actions are as important as the immediately actions in incident. The incident can be defined as an action which can negative influence flow of traffic. For example, environmental accident, traffic obstructions or planed reconstructions. The critical infrastructure defines systems and subsystems of attributes of critical infrastructure which disruptions can have negative influence to security of state. [1]

The incident may occur each of the sectors of the critical infrastructure. The implications of incident may have an impact on other elements. After the incident, we determine the severity of the impact on the people, economy, environment, public health and the psych logistical impact. Severity turned out depends on the time for which the functionality will be restored. [2] The many authors solved this problem with the different types to approval. For example, with many criterial decision making [1] or mathematical calculation of the risk of the critical incidents [3] and the last but not the least by the international standard Business Continuity Systems [4].

In this research the evaluation criteria and the term road transport will be solved by means of the fuzzy logic. The advantages of the fuzzy logic in dealing with the prevention as well as response importance, because it is not operating only the states 0 and 1 , but with the whole interval. 
When an incident is necessary to consider to the several aspects. It is not only the travel time from point A to point B. Our proposed software should funk as a tool not only for the prevention of the emergence of the incident. Software should no longer fully respond in the section at incident. This paper is organized as follows. In section 2, we defined the primary principle of fuzzy logic. This part is held on the proposal of application, defuzzification, and examples of an algorithm. Finally, we identified advantages and disadvantages of this solutions.

\section{Fuzzy logic}

The fuzzy logic is the sophisticated programming logic, which can use a lot of values for decision-making. It is not about two parameters, but it is about the whole examination range of the values. The state of the decision-making is not only OK or NOK, but the software examines each of the values and the states. Finally, the software makes a decision. It is important to implement expert's findings and translate it to fuzzy logic. It means, a definition of range for the decision support. In Figure 1 can be seen this described method.

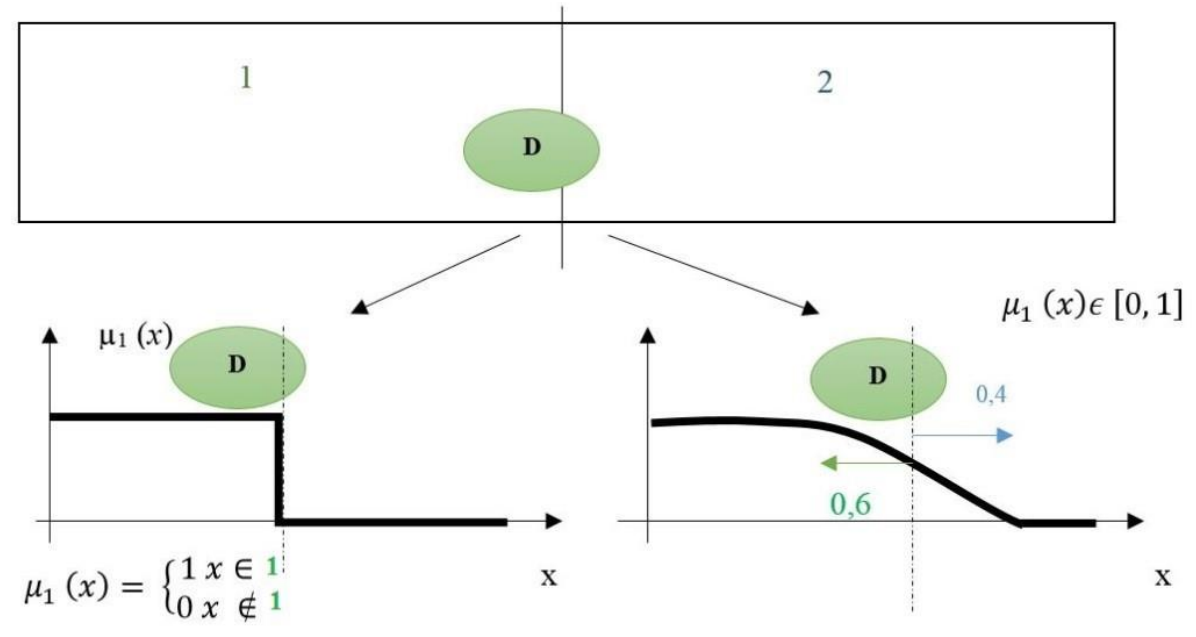

The classical theory

The fuzzy logic

Fig. 1. The differences between classical and fuzzy logic. [5]

In the classical theory, we can have two values, and it is 1 and 0 . It means, element D (Figure 1) belongs to group 1, or element D does not belong to group 1. In the other side, the fuzzy logic knows about each of the value in the interval from 0 to 1. Fuzzy Logic has special rules and symbols (operates) in mathematical operations. They are called Fuzzy statements. In fuzzy logic, we use linguistic variables, and it is defining by universal. The value of the linguistic variable we can mark as an element of plurality. [6]

Inputs are sharp values, which are in fuzzifier process transferred to fuzzy sets. With fuzzy sets, we can make special fuzzy operations, which are represented by rule base. In the end, processes reverse. We have fuzzy set, and we must define sharp value. We know more as one method for defuzzifer. For example, it is a centre of gravity or the mean of maxima. [7]

\subsection{The proposal of the application}

In the proposal of software, the fuzzifier was used in the processes when sharp values will be transformed into linguistic values. Sharp values will be gotten from inputs parameters and from law requirements too. It is a process which can define as the preparatory process. The defuzzifier is using in the process when interval must be transformed to one sharp value, which represents this solution in incidents.

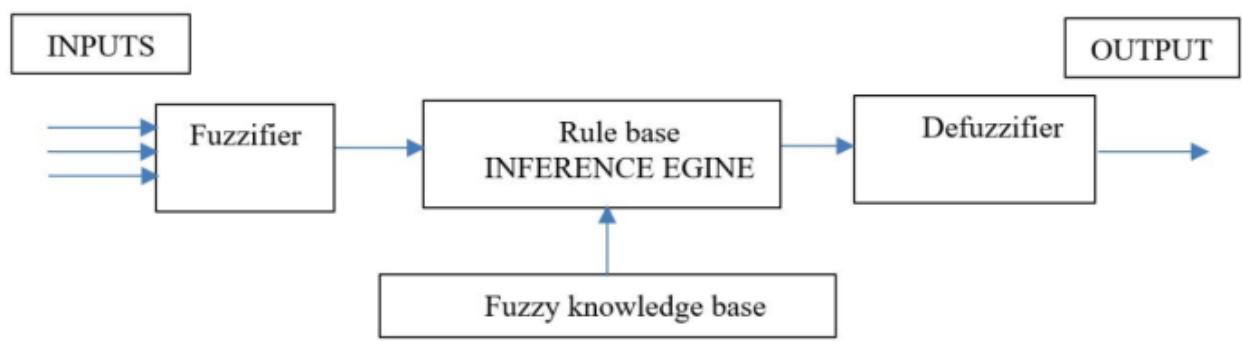

Fig. 2. The basic of fuzzyfication process. [5] 
Interpreting if-then rules a three-part process. This process is explained in detail in the next section [8]:

- Fuzzify inputs: Resolve all fuzzy statements in the antecedent to a degree of membership between 0 and 1 . If there is only one part to the antecedent, this is the degree of support for the rule.

- Apply fuzzy operator to the various part precursors: If there are multiple elements to the antecedent, use fuzzy logic operators and resolve the antecedent to a single number between 0 and 1 . This is the degree of support for the rule.

- Apply implication method: Use the degree of support for the whole rule to shape the output fuzzy set. The consequent of a fuzzy rule assigns an entire fuzzy set to the output. This fuzzy set is represented by a membership function that is chosen to indicate the qualities of the consequent. If the antecedent is only partially true, (i.e., is assigned a value less than 1), then the output fuzzy set is truncated according to the implication method.

In general, one rule by itself does not do much good. What the proposal need are two or more rules that can respond another. The output of each rule is a fuzzy set. Then the output fuzzy sets for each rule are aggregated into a single output fuzzy set. Finally, the resulting set is defuzzified or resolved to a single number [7].

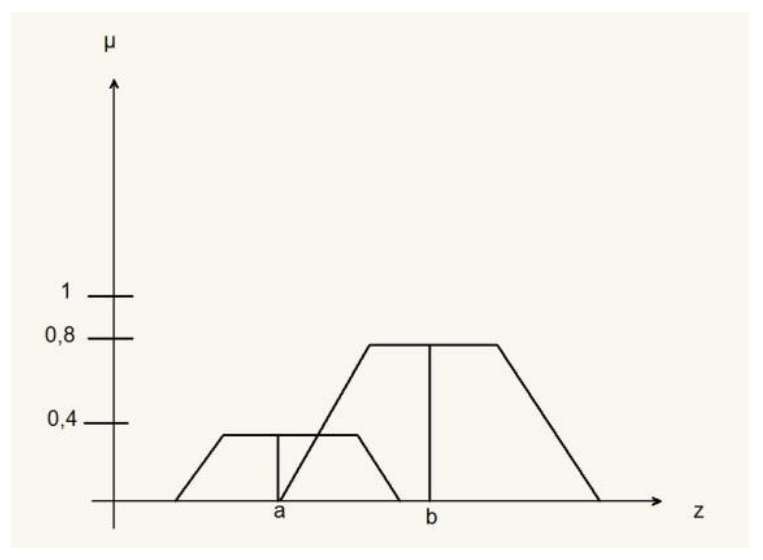

Fig. 3. Defuzzification with Weighted Average Method. [5]

The next equation formulates principle of this defuzzification method [5].

$$
z^{*}=\frac{\sum \mu_{c}(\bar{z}) \times \bar{z}}{\sum \mu_{c}(\bar{z})}
$$

In the next equation, the example of a model that is depicted in graph.

$$
z^{*}=\frac{a(0,4)+b(0,8)}{0,4+0,8}
$$

In this process, more methods, which are not described in a paper, can be used. For example, a center of gravity, height method, middle of maxima, a center of sums, a center of the largest area and the last method is first or last of maxima.

\subsection{The proposal of the application}

Fuzzy rule is in the next text: "if (fuzzy statement) and (fuzzy statement) then (fuzzy statement)". [8] If (material=dangerous) and (date=critical) then (transported road = high degree).

If (transported time $=$ high) and (material $=$ perishable foodstuffs) then (requirements $=5$ degree) and (frequency road $=$ low).

When we are working with the statement, we will use logical operators. It will describe the interrelationship between these groups of variables. In solutions, which are oriented into transport, the individual characteristics are identified. The requirements for the road and travel are so different that it is not possible to define one solution for each category. The next reason is that each transport has specific attributes. It is not possible that operator can use the binary system, but he needs to indicate a relation between the characteristics, requirements and measures. It is evident that in every transportation process is a risk, but we must determine the value, which represents this danger in each transported unit, in each time and with each material. On the other hand, when we know about law requirements, we can set up regular interval from legislative. Sub-program can be defined, which will detect limits or some range before the limit. It can be the implementation of test driver licenses and others. [9] 
We can identify next fuzzy statement:

If (planned road=detours interval 1) than (construction vehicles category $1=$ not allowed).

This fuzzy statement means that detours interval 1 represented road with a specified number of detours. We can identify more detours interval as we want. Similarly, then we can see it in construction. Construction vehicles category 1 represents vehicles with weight more than $12 \mathrm{t}$. All development of the software is the result more analyses. This proposal needs for correct function more analyses. We can tell, that this software is addicted on this inputs data. These data consider with the current state of the road, current traffic occupancy and planned information. In a category of planned in-formation data about transportation are, which we can identify with this proposal of software.

\section{Conclusion}

Road transport is the most used not only in the Czech Republic. Upon the occurrence of an incident here in the transport sector borne by the event of high suggestibility factor other elements of a critical infrastructure as was described in the previous research. [10]

From a mathematical point of view, the most appropriate way to process input data can be to use fuzzy logic. This method we can choose because every statement professional are presented by a fuzzy statement. Because of similar tools already exist, it is possible for us proposed device compared with existed tools and improve in areas where competitors are lagging behind. You cannot come up with the perfect tool, and we can record both positives and negative. The advantage of fuzzy logic is examining the entire interval and not only two states ( 0 and 1$)$.

Negatives include specific input data, the tool needs and there are a large number of instruments to be used to be. It is worth noting that even individuals have different priorities and then identify the most appropriate criteria for all can be quite challenging. Among the strengths of the proposed instrument, it is certainly of timely responses to possible situations that are such. Reconstruction, road closures or traffic accidents reported. Further strengths as a rapid response when planning routes, by category pre-ported material we can determine the most appropriate means of transport. In the last but not least, I must mention a big plus between instrument from the viewpoint of the transport sector but also the sector cybernetic.

In the future research we will apply or defined fuzzy statements to the real conditions. It means, the next research will be focused on the case study and the usability of the proposed fuzzy statements. According to the results, the fuzzy statements can be repaired.

\section{Acknowledgments}

This project is realized as the research with doctoral student and it is the basic input for next research, which we will develop in next term. It was realized with support of the university. This work was supported by Internal Grant Agency of Tomas Bata University under the project No. IGA/FAI/2018/013. This work was supported by the research project VI20152019049 "RESILIENCE 2015: Dynamic Resilience Evaluation of Interrelated Critical Infrastructure Subsystems", supported by the Ministry of the Interior of the Czech Republic in the years 2015-2019.

\section{References}

[1] M. Hromada and L. Lukáš, "Conceptual design of the resilience evaluation system of critical infrastructure elements and networks in selected areas in Czech republic", IEEE International Conference on Technologies for Homeland Security, (2012) November 13-15, Boston, USA.

[2] M. L. Garcia, "The Design and Evaluation of Physical Protection Systems", Second edition, Sandia National Laboratories, (2007), pp. 275, ISBN - 10: 0-7506-8352.

[3] M. Hyslop, "Critical Information Infrastructures, Resilience and Protection”, Springer, Middlesbrough, 1st ed., (2010) November, pp. 288, ISBN 978-1441944191. International Journal of Disaster Recovery and Business Continuity Vol. 3, November, 2012.

[4] K. J. Engeman and D. M. Henderson, "Business Continuity and Risk Management, Essentials of Organizational Resilience”, Rothstein Associates, 1st ed., (2011) September, pp. 370, ISBN 978- 1931332545.

[5] Duricova, P. L. Hromada, M. The Proposal of the Soft Targets Security. Advences in Intelligent Systems and Computing, Automation Control Theory Perspectives in Intelligent Systems. Proceedings of the 5th Computer Science On-line Conference 2016 (CSOC2016), Vol3, Springer, pp.: 337-345. ISSN 2194-5357, ISBN 978-3-31933387-8, DOI 10.1007/978-3-319-33389-2.

[6] Zadeh, L. A., 1965. Fuzzy Sets. In: Information and Control, vol. 8, 338-353.

[7] Dernoncourt, F.: Introduction to fuzzy logic, Massachutsetts Institute of Technology (2013).

[8] Rohan, 2016, If than rules. In: http://www.rohan.sdsu.edu/doc/matlab/toolbox/fuzzy/fuzzytu5.html

[9] Klir, G. J. Yuan, B.: Fuzzy Sets and Fuzzy logic. In: Theory and Application. Prentice Hall, pp. 574. New Jersey (1995).

[10] Mrazek Jan, Duricova, Lucia \& Martin Hromada. The Mathematical Modeling of Road Transport in Context of Critical Infrastructure Protection: Reviews, Vol. 1, Book Series, Barcelona, Spain, IFSA Publishing, S.L., ISBN: 978-84-697-8994-0, 2018. 